\section{Regards sur l'économie allemande}

Bulletin économique du CIRAC

$71 \mid 2005$

Varia

\title{
Politique familiale : un débat politique et sociétal
}

\author{
Marie-Hélène Pautrat
}

\section{OpenEdition}

Journals

Édition électronique

URL : http://journals.openedition.org/rea/332

DOI : $10.4000 /$ rea.332

ISBN : 978-2-8218-0838-6

ISSN : 1965-0787

Éditeur

CIRAC

Édition imprimée

Date de publication : 1 mai 2005

Pagination : 25-30

ISSN : 1156-8992

Référence électronique

Marie-Hélène Pautrat, «Politique familiale : un débat politique et sociétal », Regards sur l'économie allemande [En ligne], 71 | mai 2005, mis en ligne le 06 mai 2008, consulté le 10 décembre 2020. URL http://journals.openedition.org/rea/332 ; DOl : https://doi.org/10.4000/rea.332 


\section{Politique familiale : un débat politique et sociétal}

\section{Marie-Hélène Pautrat}

La politique familiale pourrait bien devenir un thème majeur des élections fédérales de 2006 : conséquence du débat sur l'évolution démographique du pays, l'Allemagne s'interroge sur les causes de son déficit de naissances. L'héritage historique ayant profondément et durablement porté le discrédit sur toute politique "nataliste", les mesures financières, généreuses mais disparates, se sont multipliées sans pour autant se révéler suffisantes si on en juge aux taux de fécondité très bas depuis trente ans. Aujourd'hui, la dimension économique d'un déficit durable de naissances est évidente et contraint les pouvoirs publics à décréter l'urgence. D'autant que le contexte sociétal et les débats, notamment depuis l'unification, sur les formes diversifiées de la famille, l'émancipation économique des femmes ou encore l'efficacité du système éducatif, remettent en question des valeurs fortement ancrées dans la société (ouest-) allemande. Promouvoir le travail féminin et le potentiel humain qu'il représente sans condamner les perspectives démographiques du pays : tel est l'objectif des mesures qui entrent en vigueur en 2005, au nom à la fois de l'équité des chances et de la liberté pour les femmes de concilier vie familiale et professionnelle. Sauront-elles constituer un signal positif ? Rien n'est moins sûr. L'aspect financier et la question de la garde d'enfants, bien qu'importants, ne constituent pas à eux seuls des clés suffisantes. L'environnement culturel et la capacité d'une société à faire vivre ensemble toutes les générations constituent sans doute le véritable enjeu.

En 2004, l'Allemagne a enregistré 712000 naissances, un chiffre qui continue de décroître depuis 1991 (à l'exception de 1996 et 1997), et a pratiquement diminué de moitié en quarante ans (1964:1,35 millions). Avec une nuance toutefois : après s'être effondré dans les Länder orientaux au lendemain de l'unification, le nombre de naissances y progresse à nouveau depuis 1995. Le taux de fécondité qui, depuis le milieu des années 1970, fluctue entre 1,3 et 1,4 enfant par femme, se situe bien en deçà du seuil de renouvellement des générations $(2,1)$ et demeure parmi les plus bas en Europe, avec l'Italie, l'Espagne et la Grèce. Cette tendance est imputable à la part croissante des personnes sans enfants. C'est le cas d'un tiers des femmes nées en 1965, chiffre probablement plus élevé parmi les diplômées du supérieur (du moins à l'ouest) mais que les statistiques allemandes ne permettent pas de saisir avec précision. Tout aussi révélateur, le désir d'enfants chute également, selon les enquêtes de l'institut de recherche démographique BIB : avec 1,52 enfant souhaité par femme entre 18 et 39 ans (2,25 en France), l'Allemagne partage là aussi les dernières places en Europe, avec l'Autriche et l'Italie.

Les progrès de la contraception, la hausse du niveau de formation des femmes pour une durée des études supérieures plus élevée en Allemagne, l'âge moyen des femmes à la naissance du premier enfant (2003 : 29,9 ans), sont autant de facteurs explicatifs de la baisse du nombre de naissances. L'aspiration des femmes à l'indépendance économique et leur participation au marché du travail sont confirmées par l'augmentation continue du taux d'activité des femmes de 15 à 65 ans: il frôlait en 2002 l'objectif de $60 \%$ affiché par l'Agenda de
Un déficit de naissances qui perdure depuis 30 ans

Le taux d'emploi féminin progresse 
Mesures financières : clef de voûte de la politique familiale allemande

Des mesures fiscales en faveur des familles...

... complétées par le dernier volet de la réforme fiscale

Des prestations familiales généreuses
Lisbonne $(59,1 \%$; ouest : $58,8 \%$, est : $71,9 \%)$; les femmes représentaient $46 \%$ des actifs (17,9 millions) en 2003. L'emploi féminin y est d'ailleurs explicitement considéré comme un potentiel indispensable pour l'activité économique et la pérennisation des systèmes sociaux : comme réponse possible aux pénuries de main d'œuvre attendues dans certains secteurs, mais aussi pour élargir l'assiette de cotisation et relever le niveau de pension des femmes (voir REA 70/05). C'est en ce sens que la politique européenne de l'emploi et, plus spécifiquement pour l'Allemagne, les recommandations de la commission Hartz et l'Agenda 2010, plaident pour une politique incitant les parents à ne pas quitter leur emploi pour s'occuper des enfants. Les études comparatives en Europe établissent d'ailleurs que forte participation des femmes au marché du travail et taux de fécondité élevés ne sont pas contradictoires, bien au contraire (voir l'Islande, la France, les pays scandinaves). La conciliation entre vie familiale et professionnelle constitue dès lors la priorité pour le gouvernement fédéral dans ses orientations de politique familiale.

Les mesures en faveur de la famille sont loin d'être inexistantes, puisque l'Allemagne y consacre selon l'OCDE $2,3 \%$ de son revenu national, soit près de 150 milliards $€$ (2000) d'après les estimations de la Bundesbank. La Loi fondamentale (article 6) place explicitement la famille (et le mariage) sous la protection particulière de l'Etat. Mais la politique familiale se distingue essentiellement par son volet financier, les prestations versées aux familles comptant parmi les plus élevées en Europe. Elles reposent sur un système complexe, régi pour l'essentiel par la Loi sur l'impôt sur le revenu et la Loi fédérale sur les allocations familiales, qui imbrique étroitement aides directes (sous forme de prestations familiales) et avantages fiscaux. Ce dispositif a d'ailleurs fait l'objet de plusieurs corrections ces dernières années, notamment sous l'injonction du Tribunal constitutionnel fédéral invitant le législateur, au nom d'un impératif d'égalité fiscale et de compensation des charges familiales, à corriger certaines dispositions défavorisant les parents par rapport aux contribuables sans enfant ou les couples mariés par rapport aux parents isolés.

Le régime fiscal allemand prévoit aujourd'hui un abattement fiscal forfaitaire pour enfant à charge (Kinderfreibetrag) de $3648 €$ par an et foyer fiscal pour les couples (moitié moins pour un parent isolé), ainsi qu'un abattement supplémentaire de $2160 €$ (1 $080 €$ pour un parent isolé) par an et enfant de moins de 16 ans au titre de la charge éducative (Betreuungsbetrag). Et c'est là qu'intervient l'imbrication avec le versement des prestations directes : en effet, ces abattements sont acquittés en Allemagne par le versement des allocations familiales. Ce n'est que lorsque les prestations familiales sont inférieures au montant généré par les abattements fiscaux que ces derniers sont pris en compte. Sont donc concernés les revenus les plus élevés, à charge pour l'administration fiscale de déterminer le régime le plus avantageux pour chaque famille. Enfin, un abattement supplémentaire de $1500 €$ pour les enfants de moins de 14 ans a été introduit en 2002 au titre des services de garde d'enfants.

L'entrée en vigueur du troisième (et dernier) volet de la réforme fiscale au $1^{\mathrm{er}}$ janvier 2005 conduit par ailleurs à une baisse de l'impôt sur le revenu qui doit bénéficier plus particulièrement aux familles aux revenus les plus faibles: le taux d'imposition de base vient ainsi d'être abaissé à $15 \%$, contre $26 \%$ en 1998. Le plancher d'imposition est pour sa part rehaussé, passant de $6322 €$ à $7664 €$. Les parents isolés bénéficient par ailleurs de mesures spécifiques. La fiscalité constitue donc un aspect important de la politique familiale, avec toutefois un bémol, mais d'importance : les époux peuvent opter pour une imposition conjointe (Ehegattensplitting) dont le calcul génère un allègement fiscal d'autant plus important pour les foyers avec un seul salaire. A cet égard, le régime fiscal reste peu incitatif pour l'emploi des mères.

En ce qui concerne les aides directes, les allocations familiales (Kindergeld), financées par l'impôt conjointement par le Bund (à 76 \%) et les Länder, sont 
versées sans condition de ressources sous forme de bonification fiscale à un membre désigné du foyer ou, dans le cas de parents isolés, à la personne ayant la garde de l'enfant. Augmenté à trois reprises depuis 1999 par le gouvernement Schröder, leur montant s'élève depuis le $1^{\mathrm{er}}$ janvier 2002 à $154 €$ par mois pour chacun des trois premiers enfants, puis à $179 €$ pour le quatrième enfant et les suivants, jusqu'à l'âge de 18 ans. Mais les allocations peuvent être versées jusqu'à 27 ans lorsque les enfants suivent une formation ou 21 ans en cas de recherche d'emploi. Dans tous les cas, les versements cessent dès lors que les revenus de l'enfant majeur atteignent la somme de $7680 €$.

Enfin, le gouvernement Schröder vient d'introduire au $1^{\mathrm{er}}$ janvier 2005 , et pour une durée maximale de 36 mois, une majoration complémentaire (Kinderzuschlag) pour les enfants mineurs, pouvant atteindre $140 €$ par mois. Cette prestation familiale, réglementée par la loi fédérale sur les allocations familiales, intervient dans le sillage de la Loi Hartz IV. Selon les propos de la ministre de la Famille Renate Schmidt (SPD), il s'agit d'apporter un soutien aux parents qui travaillent pour les aider à subvenir aux besoins de leurs enfants. Elle cible dès lors les revenus modestes, en particulier les mères actives aux revenus les plus faibles et n'est donc destinée ni aux bénéficiaires de l'aide sociale, ni à ceux de la nouvelle « allocation chômage II » (Arbeitslosengeld II). Selon Michael Fertig de l'institut de recherche économique RWI (Handelsblatt, 02-03-2005), cette mesure est en fait censée "empêcher une augmentation de la pauvreté chez les enfants ", comme conséquence possible de l'introduction de la Loi Hartz IV.

Bien que globalement généreux, le système allemand de prestations financières montre ses limites au regard de l'augmentation continue, durant les années 1990, du taux de pauvreté chez les enfants. Selon une étude réalisée par I'UNICEF en collaboration avec le RWI et présentée début mars, la pauvreté touche aujourd'hui 10,2\% des enfants en Allemagne, malgré un niveau de prestations sociales sensiblement équivalent au plan financier à celui des pays scandinaves, dont le système social permet toutefois de lutter plus efficacement contre la pauvreté chez les enfants. Première cause de cette piètre performance : la nature des prestations, insuffisamment ciblées et saupoudrées indifféremment entre familles nécessiteuses et familles aisées. Mais également, et contrairement encore aux pays scandinaves ou à la France, l'absence d'une offre étendue d'infrastructures de garde d'enfants s'avère un frein puissant à l'accès au marché du travail des femmes. L'expérience des Länder orientaux viendrait confirmer cette hypothèse : la pauvreté chez les enfants s'y développe moins rapidement qu'à l'ouest en dépit de revenus globalement inférieurs et d'un chômage beaucoup plus élevé. La participation des mères au marché du travail y est plus développée, de même que les possibilités de garde. II s'agit là d'un point-clef, sachant que la pauvreté chez les enfants se concentre sur les foyers de chômeurs, les familles immigrées et, de plus en plus, sur les familles monoparentales : $3 \%$ des couples sont concernés contre $40 \%$ des parents isolés, et $84 \%$ des 2,4 millions de personnes élevant seules leurs enfants sont des femmes, selon les données du ministère de la Famille (BMFSFJ).

\section{La réforme du congé parental de 2001}

Le congé parental (Elternzeit) permet aux parents exerçant une activité de prendre en charge leur enfant jusqu'à ses 3 ans, une partie du congé parental pouvant être reportée jusqu'au $8^{\mathrm{e}}$ anniversaire. La réforme de 2001 aménage par ailleurs le droit, pour les salariés d'entreprises de plus de 15 personnes, d'exercer un emploi à temps partiel (de 15 à 30 heures par semaine), Cette réduction du temps de travail peut bénéficier simultanément aux deux parents, qui cumulent dès lors jusqu'à 60 heures de travail hebdomadaires à eux deux au sein du foyer. Cette réforme est une première entorse au modèle familial traditionnel de 'père nourricier', aux effets limités, il est vrai : $2 \%$ seulement des pères bénéficiaient en 2002 de ce congé parental, selon les données du BMFSFJ.

Autre volet majeur du soutien financier aux familles, l'allocation parentale d'éducation (Erziehungsgeld) est accordée par le gouvernement fédéral aux termes de la loi fédérale amendée en 2001 ; elle s'applique pour les enfants nés à
Introduction d'une majoration complémentaire en 2005

Un système généreux de prestation, mais peu efficace

L'allocation parentale d'éducation : évolution à la marge... 
... ou amorce d'un changement de paradigme?

L'infléchissement de la politique familiale

La garde des enfants : un véritable nœud gordien dater du $1^{\text {er }}$ janvier 2001 et est versée sous condition de ressources jusqu'aux 24 mois de l'enfant gardé par ses parents s'ils ne travaillent pas plus de 30 heures hebdomadaires. Le montant de l'allocation s'élève jusqu'à $300 €$ par mois, à moins que les parents n'optent pour un versement sur 12 mois (maximum : $450 €$ mensuels). Le plafond du revenu annuel net a été abaissé à $30000 €$ pour le premier enfant jusqu'à ses 6 mois, et à $23000 €$ pour un parent isolé. II s'abaisse à partir du $7^{\mathrm{e}}$ mois de l'enfant, à $16500 €$ pour obtenir la totalité de l'allocation (parent isolé : 13450 ) à $22086 €$ pour recevoir une allocation partielle. Pour chaque enfant supplémentaire dans le foyer, les différents plafonds augmentent de $3140 €$.

Mais d'ores et déjà, un nouveau concept de réforme se dessine pour 2006 qui, s'il est adopté, annoncerait un véritable changement de paradigme dans la conception même des prestations familiales : l'allocation parentale serait alors versée pour une année seulement, afin d'accélérer le retour des mères dans l'emploi : en effet, $40 \%$ des femmes ne reprennent pas leur travail à l'issue de leur congé parental. Mais surtout, elle s'alignerait sur l'indemnité chômage, soit $67 \%$ du salaire antérieur. Cette mesure, d'autant plus attractive pour les revenus élevés, s'adresse donc explicitement aux femmes diplômées qui hésitent à compromettre une carrière professionnelle le temps de la maternité. Les arguments avancés pour justifier cette mesure relèvent d'un calcul simple : l'Etat, par les transferts financiers, prendrait en charge l'essentiel du coût de l'éducation pour les foyers aux revenus modestes, ce qui poserait un problème d'équité vis-à-vis des ménages aux revenus plus élevés qui consacrent une part beaucoup plus importante de leur revenu à l'éducation des enfants. Par ailleurs, l'étude PISA (Programme for International Student Assessment) de l'OCDE (voir REA 66/04), point d'ancrage de la plupart des débats sociétaux en cours, a, il est vrai, établi une forte corrélation entre le niveau de qualification des parents et la réussite scolaire des enfants en Allemagne. Rien ne permet pour autant d'en déduire que le niveau d'éducation des parents et le statut social se révèlent plus déterminants que les conditions d'éducation offertes aux élèves, et cette conception est très controversée. Mais l'argument suit son chemin, dans un pays qui considère l'investissement dans le capital humain comme son principal atout pour l'avenir. L'allocation parentale perdrait donc toute dimension sociale pour devenir expressément une incitation pour les femmes diplômées.

En soi, cette reprise du débat par la classe politique allemande est loin d'être anodine dans un pays où la notion de politique nataliste a été rendue taboue, d'une part par son instrumentalisation par le régime hitlérien et sa main mise sur l'éducation des jeunes, d'autre part par le rejet du modèle est-allemand d'organisation sociale favorisant pour des motifs économiques et idéologiques le travail féminin et la prise en charge des enfants par les structures étatiques. Pour des raisons culturelles aussi, la société allemande contemporaine a fini par stigmatiser l'image de la mère qui travaille et développer de très fortes réticences sur les questions de socialisation précoce. Or, ce modèle social ne répond aujourd'hui ni aux aspirations de la société, ni aux réalités socioéconomiques. Promouvoir un renversement des mentalités progressif par de nouvelles orientations politiques est le pari que tente de relever depuis 2004 le gouvernement en axant ses efforts sur deux priorités : l'accueil des enfants et plus généralement la conciliation entre vie familiale et professionnelle, en quelque sorte en promouvant une politique familiale 'moderne' destinée moins à soutenir la famille qu'à permettre l'activité professionnelle des mères sans condamner les perspectives démographiques.

En matière de structures d'accueil de l'enfance, l'Allemagne affiche à la fois une longue tradition (depuis le $\mathrm{XIX}^{\mathrm{e}}$ siècle) et un fort déficit quantitatif. Ce dernier constat mérite néanmoins quelques nuances, au moins en fonction de deux aspects : les classes d'âge accueillies, et les disparités entre Länder orientaux et occidentaux. La loi du $1^{\text {er }}$ janvier 1999 qui octroie à chaque enfant dès trois ans et jusqu'à son entrée dans le système scolaire le droit à une place dans un jar- 
din d'enfant a sensiblement fait évoluer l'offre au cours des dernières années. L'Allemagne comptait ainsi, fin 2002, 2507744 places (ouest : 2088176 ; est : 341328 ), pour 2,7 millions d'enfants de cette classe d'âge, couvrant ainsi $90 \%$ environ de la demande, du moins à un plan purement quantitatif.

C'est donc au niveau des structures d'accueil de la petite enfance que le déficit reste le plus criant. Fin 2002, l'Allemagne comptait 190395 places de crèche (mais seulement 50775 à l'ouest) pour 2,23 millions d'enfants de moins de trois ans, permettant la prise en charge de $35 \%$ de cette tranche d'âge à l'est contre seulement 2,5\% à l'ouest (objectif européen fixé lors du sommet de Barcelone : $33 \%$ ). La loi portant sur le développement des capacités de garde (Tagesbetreuungsausbaugesetz), adoptée par le parlement à l'automne 2004 malgré l'opposition des Länder CDU et CSU, prévoit donc de programmer entre 2005 et 2010 le rattrapage, en s'appuyant sur une offre diversifiée : 230000 places supplémentaires doivent en principe être créées pour les moins de trois ans, dont un tiers auprès d'assistantes maternelles. Le financement incombera aux communes et aux Länder, compétents pour l'organisation de la garde d'enfants, à partir des économies réalisées par les communes par la modification du régime de l'aide sociale dans le cadre de la loi Hartz IV : 1,5 milliard € par an pourraient être dégagés à cet effet. Or, selon l'institut berlinois DIW, ces mesures resteraient en deça de la demande effective : elles n'apportent pas de perspectives tangibles pour les mères souhaitant (re)travailler (estimations DIW : 1,2 million de places nécessaires). Par ailleurs, une partie importante des ressources dégagées par la loi Hartz IV devrait mécaniquement revenir aux communes est-allemandes, bien mieux loties en matière d'accueil des enfants.

Le rattrapage doit être également qualitatif : en particulier, il doit proposer une offre de qualité (ce qui implique d'investir massivement dans la qualification des personnels en charge de la petite enfance, de mettre en place un dispositif de contrôle de la part des autorités), et adaptée aux besoins exprimés. Or, les rythmes d'ouverture des institutions restent en déphasage par rapport aux contraintes de la vie professionnelle (horaires atypiques, flexibles, voire imprévisibles, temps de transport accrus, etc.). Dans les anciens Länder (ce n'est pas le cas à l'est), la plupart des jardins d'enfants ferment à midi et permettent difficilement à la mère (dans la grande majorité des cas) d'occuper un emploi, sauf à temps très partiel. Cette situation ne manque pas de soulever une nouvelle fois la question de la socialisation précoce, mais aussi du développement des capacités cognitives et linguistiques de l'enfant dans le cadre du système éducatif. La diffusion progressive du concept d'école toute la journée (la Ganztagsschule), financée par une subvention du Bund aux Länder (4 milliards $€$ d'ici 2007) dans le cadre de l'Agenda 2010, a été une première forme de réponse aux résultats des études comparatives internationales (PISA, IGLU sur la lecture en primaire, etc.). L'accueil de la petite enfance pour permettre un développement du travail féminin compatible avec la vie familiale reste un chantier à part entière.

On peut toutefois légitimement se demander si les causes du déficit de naissances relèvent simplement du niveau de prestations ou de l'insuffisance des infrastructures d'accueil, quand le problème touche notamment les femmes les plus qualifiées. Comme l'a diagnostiqué Renate Köcher, directrice de l'Institut de démoscopie d'Allensbach, on assiste à une "distanciation croissante » dans la société allemande car des groupes sociaux entiers finissent par perdre le contact avec les enfants (Frankfurter Allgemeine Zeitung, 12-01-2005). Le gouvernement s'est donc fixé comme priorité de sensibiliser la sphère professionnelle et de renforcer la coopération avec les acteurs économiques et sociaux. Les entreprises (tout au moins par la voix des fédérations professionnelles) commencent en effet à s'intéresser à la question de la conciliation entre vie familiale et professionnelle, les évolutions démographiques penchant en faveur d'un renforcement de l'emploi féminin. Des initiatives variées voient le jour, avec en commun l'objectif de générer un 'climat positif' pour les familles dans la
Une priorité du gouvernement : l'accueil des moins de trois ans

Des structures d'accueil qui doivent répondre aux besoins

Renforcer la coopération avec les acteurs économiques et sociaux 
Une «Alliance pour la famille» pour repenser les politiques d'entreprise

société allemande. Les «pactes locaux pour la famille » (131 réseaux aujourd'hui), lancés en 2004 par le gouvernement fédéral, visent ainsi à impliquer communes, entreprises, églises ou associations (qui gèrent nombre de jardins d'enfants), etc. pour générer de nouveaux projets, notamment en matière de garde des enfants, en fonction des besoins exprimés au niveau local (www.lokale-buendnisse-fuer-familie.de).

Lancée en 2003 par le BMFSFJ, la fondation Bertelsmann, des entreprises et les partenaires sociaux, l'initiative Allianz für Familie entend par ailleurs faire évoluer les organisations du travail et les cultures d'entreprises, mais aussi inciter à inclure dans les conventions collectives des dispositions prenant en compte vie professionnelle et familiale. Le temps partiel, très développé chez les mères, ne saurait suffire : il réduit les perspectives professionnelles (faible représentation des femmes dans les postes de management), demeure un facteur de paupérisation et a des effets néfastes sur les systèmes de protection sociale. D'autres modèles doivent donc essaimer: télétravail, Job-Sharing, éventuellement crèches d'entreprises malgré des réticences (moins de $2 \%$ des entreprises disposeraient de leur crèche, pour des raisons de coût, d'organisation, mais aussi de demande mal identifiée), congés sabbatiques ou, plus fondamentalement, l'organisation dans la durée des carrières féminines. Enfin, un atlas de la famille (Familienatlas 2005), publié en janvier 2005 par l'institut Prognos AG en partenariat avec l'hebdomadaire Die Zeit, entend susciter une prise de conscience à tous les échelons : en s'intéressant directement aux villes, c'est-à-dire à la vie et l'action publique locales, il oppose très concrètement niveau de vie actuel et perspectives démographiques.

RECUL DU NOMBRE DE NAISSANCES DEPUIS 30 ANS, hausse de la participation, à la fois souhaitée et nécessaire, des femmes au marché du travail, débat moins crispé sur l'apport de la société dans la construction du jeune enfant : c'est sur la base de cette triple évolution que le gouvernement allemand tente de donner de nouvelles orientations à la politique familiale allemande. Acteurs politiques et économiques s'entendent aujourd'hui pour replacer la famille au centre du débat socio-politique, et en particulier soutenir les mères qui travaillent. La Familienfreundlichkeit, terme spécifique désignant l'attention portée à la famille, passe aujourd'hui pour un avantage compétitif. Pour autant, le discours doit encore essaimer et se traduire dans les faits. On peut par ailleurs douter de la portée des nouvelles mesures, alors que les réponses sont à chercher dans une évolution radicale des mentalités, certes en phase avec les aspirations des mères, mais pas forcément avec les habitudes et comportements collectifs. Et il n'est pas évident que la mise en avant d'arguments économiques soit suffisante quand le débat relève avant tout de la sphère privée et que ce sont des valeurs et un modèle d'organisation qu'il convient aujourd'hui de réinterroger.

\section{Indications bibliographiques}

\footnotetext{
Bundesministerium für Familie, Senioren, Frauen Und Jugend, Frauen in Deutschland, BMFSFJ, Berlin, 2004

Bundesministerium für Familie, Senioren, Frauen und Jugend / Deutsches JugendINSTITUT, OECD Early Childhood Policy Review 2002-2004. Hintergrundbericht Deutschland, DJI, Munich, 2004

«Brauchen wir eine neue Familienpolitik? Zeitgespräch mit Beiträgen von Josef Schmid, Notburga Ott, Stefan Sell sowie Thomas Bahle », Wirtschaftsdienst, n³, mars 2005

Corak M., Fertig M., TAmm M., A portrait of Child Poverty in Germany, RWI Discussion Paper, n²6, RWI, Essen, 2005

FAGNANI J., "Schwestern oder entfernte Kusinen ? Deutsche und französische Familienpolitik im Vergleich ", in Neumann W. (ed.), Welche Zukunft für den Sozialstaat? Reformpolitik in Frankreich und Deutschland, VS Verlag für Sozialwissenschaften, 2004

SPIESS K., "Wie viele Kinderbetreuungsplätze fehlen in Deutschland ?: Neue Bedarfsermittlung für Kinder unter drei Jahren auf der Basis von Mikrodaten », DIW-Wochenbericht $14 / 2005$
} 